\title{
INTEGRACIÓN DE PROCESOS DE NEGOCIO APLICANDO LA ARQUITECTURA ORIENTADA A SERVICIOS (SOA)
}

\author{
Marleny Peralta Ascue \\ marlenyperaltaascue@gmail.com \\ Universidad Nacional Micaela Bastidas de Apurímac, Perú \\ José Luis Merma Aroni \\ jlasesor@gmail.com \\ Universidad Nacional Micaela Bastidas de Apurímac, Perú \\ Yhon Fuentes Huamán \\ jhonf_2105@hotmail.com \\ Universidad Nacional Micaela Bastidas de Apurímac, Perú
}

Resumen

Este artículo tiene como objetivo integrar procesos de negocio en el ámbito de las pequeñas y medianas empresas (pymes), a través del desarrollo de aplicaciones web basadas en arquitecturas de integración, como la Arquitectura Orientada a Servicios (SOA, por sus siglas en inglés) y servicios web. La integración de procesos de negocio permite a las empresas unir objetivos de negocio, así como optimizar los procesos basados en el concepto de servicio. Para lograr la comunicación entre las empresas y sus clientes por medio de los servicios, se utilizaron diversos estándares web que incluyeron: XML como formato estándar para describir los datos; SOAP como protocolo de intercambio de datos; WSDL para la descripción de los servicios disponibles; y finalmente UDDI para publicar la información y comprobar qué servicios web están disponibles.

Palabras clave: procesos de negocio / aplicaciones web / arquitectura orientada a servicios / servicios web / estándares web

\section{Abstract}

\section{Integration of business processes applying service-oriented architecture (SOA)}

This article aims to integrate business processes in the field of small and medium-sized enterprises (SMEs, or PYMES for its acronym in Spanish). The integration will be achieved through the development of web applications based on integration architectures, such as Service Oriented Architecture (SOA) and Web services. The integration of business processes allows companies to join their business objectives, as well as optimize processes based on the concept of service. To achieve an optimal communication between companies and their clients through the services, various web standards were used, including: XML as standard format to describe the data; SOAP as data exchange protocol; WSDL to describe available services; and finally UDDI to publish information and check what web services are available.

Keywords: business processes / web applications / service oriented architecture / web services / web standards 


\section{Introducción}

En la actualidad, las empresas requieren de arquitecturas de software que provean a los negocios de una mayor agilidad y flexibilidad ante nuevos requerimientos; que sirvan de medio de comunicación entre grupos de personas separadas en forma organizacional, geográfica o temporal, para trabajar cooperativa y productivamente con el fin de resolver un problema que les sería muy difícil abordar en forma aislada (Ochoa, Bastarrica y Gutiérrez, 2009).

Según Bazán (2009), la Arquitectura Orientada a Servicios (SOA) es un enfoque para "el diseño y construcción de sistemas que sean flexibles y adaptables a apoyar un entorno empresarial dinámico" (p. 10). La mencionada arquitectura permite diseñar, construir, desplegar e integrar los servicios, independientemente del lenguaje en que se encuentren codificados, y de sus plataformas de ejecución. Estos servicios se encuentran interrelacionados y se definen a través de procesos de negocio conformando servicios compuestos que realizan las funciones empresariales (Bazán, 2009).

En opinión de Caicedo, Bustos y Rojas (2008), "en los últimos años ha crecido el número de desarrollos basados en el uso de servicios de software y servicios web" (p. 177). Estos cambian la forma tradicional de desarrollo, permitiendo construir aplicaciones a partir de componentes reducidos y que son configurados como servicios (Caicedo et al., 2008).

Un servicio de negocio se encuentra basado en una estructura que consta de dos partes: una que realiza las solicitudes de servicio (consumidor de servicios) y otra que representa el proveedor de servicios, es decir, el que recibe las peticiones para luego procesarlas y brindar las respectivas respuestas. Todo este proceso se ejecuta de forma ordenada a través del paso de mensajes entre el solicitante y el proveedor de servicios (Caicedo et al., 2008).

Este artículo presenta el desarrollo de dos aplicaciones web basadas en la arquitectura orientada a servicios y la implementación de un servicio web, para que dos pequeñas empresas de la región Apurímac, tomadas como referencia a criterio del investigador, puedan integrar procesos de negocio; una dedicada al hospedaje de personas como un hotel, identificada como el proveedor de servicio, y la otra empresa es una agencia de viajes, identificada como el consumidor de servicios. Se hicieron las respectivas simulaciones para comprobar la integración de sus procesos de negocio.

En el proveedor del servicio se define la descripción abstracta de ese servicio utilizando un lenguaje de descripción de servicios web (WSDL: Web Service Definition Language). Luego se creó un servicio concreto a partir de la descripción abstracta del servicio que se encarga de gestionar las reservaciones de hospedaje, registro de clientes y generar reportes, produciendo así una descripción concreta del servicio en WSDL. Esta descripción concreta se publica en un servicio de registro como UDDI (Universal Description, Discovery and Integration). Así, los consumidores y proveedores se comunican con el protocolo de mensajes 
SOAP (Simple Object Access Protocol), escrito en XML (Extensible Markup Language) y utilizado por los consumidores para enviar solicitudes, así como por los proveedores para enviar las respectivas respuestas.

\subsection{Antecedentes}

Veintimilla, Espinoza y Cisneros (2014) efectuaron un análisis del marco tecnológico relacionado con la integración de sistemas informáticos legados, revisando técnicas para la exposición de servicios, con la finalidad de establecer una arquitectura orientada a servicios que permita la integración del Programa para el Manejo del Agua y Suelo a diversos sistemas informáticos. Como resultado del estudio, concluyeron que no fue factible la integración de los sistemas empresariales, ya que ese programa "no disponía de una arquitectura robusta y madura que soporte la utilización de un bus empresarial que permita el intercambio de las diferentes aplicaciones" (p. 18).

Picón, Fontana y Martín (2014) analizaron la relación entre los servicios web y los procesos de negocio, y propusieron un modelo que hace posible la integración de procesos de negocio mediante servicios web de una manera ágil y práctica que facilita su implementación. El modelo propuesto se aplicó en el ámbito de las pequeñas y medianas empresas (pymes) para evaluar su eficiencia y determinar sus beneficios y posibles oportunidades de mejora; con ello se comprobó que un servicio web "se puede usar como la implementación de una actividad dentro de un proceso de negocio, y que un proceso a su vez puede exteriorizarse como servicio web" (Picón et al., 2014).

San José (2016) desarrolló un sistema en el que todas las funcionalidades se engloban en un único sistema, centrándose en el desarrollo de una plataforma logística, por medio del cual las empresas controlan los procesos de sus productos durante el ciclo de vida. "En cualquier punto de la cadena de suministro y automatizando o dando soporte a la toma de determinadas decisiones dentro de la empresa" (p. v). Los resultados muestran que se da uso en la comunicación entre empresas, agilizando el proceso al implementar un modelo de comunicación común, mejorando sus prestaciones de operatividad y competitividad (San José, 2016).

Caicedo et al. (2008) realizaron un estudio descriptivo de la arquitectura de desarrollo de software orientada a servicios, y entre sus principales conclusiones figura que la SOA permite la integración de diferentes tecnologías, "buscando interoperabilidad, calidad, control, eficiencia y seguridad" (p. 182), que brindan una solución "para construir sistemas que independizan la tecnología del servicio" (p. 182). Ello se puede lograr mediante una adecuada ejecución de un plan estratégico que alinee los recursos de las tecnologías de información con los objetivos de negocio, lo que permitirá una adecuada integración con los clientes y proveedores (Caicedo et al., 2008). 


\subsection{Planteamiento del problema}

Actualmente las pequeñas y medianas empresas de la región Apurímac se convierten, cada vez más, en dependientes de aplicaciones informáticas, mediante la automatización de sus procesos de negocio, los cuales deben responder rápidamente a requerimientos funcionales cada vez más cambiantes. Todas las empresas buscan optimizar procesos, incrementar su productividad y aumentar su rentabilidad; por esta razón, si se crean alianzas entre empresas se pueden unir y lograr objetivos de negocio comunes. La problemática que enfrentan las empresas de esta región es que cuentan con modelos de gestión y aplicaciones informáticas tradicionales que se orientan solo en el producto y en su área de negocio. Por este motivo, es necesario enfocar a una gestión orientada a procesos y servicios que permita la integración de nuevas empresas a través de sus procesos de negocio y, de esta manera, ofrecer productos más elaboradoso desarrollados, y extender su línea de negocio utilizando nuevas tecnologías, como la Arquitectura Orientada a Servicios (SOA), para dar soporte a sus procesos.

\subsection{Objetivos}

\subsubsection{Objetivo general}

Desarrollar aplicaciones web basadas en la Arquitectura Orientada a Servicios (SOA), para integrar procesos de negocio entre pequeñas empresas de la región Apurímac.

\subsubsection{Objetivos específicos}

Para el logro del objetivo general se formulan los siguientes objetivos específicos:

i. Analizar e identificar los procesos de negocio para declararlos como servicios.

ii. Desarrollar la aplicación web del consumidor de servicios.

iii. Desarrollar la aplicación web del proveedor de servicios.

iv. Construir un servicio web con todas las funcionalidades descritas por un servicio.

\section{Conceptos preliminares}

\subsection{Servicio web}

Con relación a los servicios web, López, Echevarría, Fierro y Jeder (2007) manifiestan lo siguiente:

es una pieza de software que conforma una serie de estándares de intercambio de información. Estos estándares permiten el intercambio de operaciones [...], 
apartándose del problema del hardware que utilicen, [y] [...] de los sistemas [...], o de los lenguajes de programación en los que estén escritos. (p. 559)

Además, haciendo referencia a Erl (2005), señalan que los web services, para mantener su independencia, encapsulan la lógica dentro de un contexto, el cual puede ser una tarea o una entidad de negocio u otra agrupación lógica (López et al., 2007).

A continuación se presentan los principales estándares empleados en el modelo de despliegue del servicio web:

i. XML (eXtensible Markup Language) estándar para registrar, transportar e intercambiar datos.

ii. SOAP (Simple Object Access Protocol - Service Oriented Access Protocol) es un protocolo que permite a los programas comunicarse vía http, independiente de plataforma y lenguaje.

iii. WSDL (Web Service Description Language) es un lenguaje basado en XML, que se usa para definir los servicios web y describir cómo acceder a estos.

iv. UDDI (Descripción, Descubrimiento e Integración Universal, en inglés Universal Description, Discovery and Integration) es un servicio de directorio donde se registran y buscan servicios web construidos por distintos proveedores. Se trata de un registro público donde se pueden realizar consultas sobre los servicios y su ubicación.

\subsubsection{Características de los servicios web}

Las características deseables de un servicio web, según Alfonso (2012), son las siguientes:

i. Accesible a través de la web, utilizando protocolos de transporte estándares y codificación de mensajes en un lenguaje estándar.

ii. Contener una descripción de sí mismo, para que se explicite la función e interfaz de dicho servicio web y sea utilizado automáticamente por cualquier aplicación.

iii. Ser localizable, es decir disponer de un mecanismo que permita ubicar un servicio web que realice una función requerida, sin que se necesite un conocimiento previo del usuario (Alfonso, 2012, p. 5).

\subsubsection{Tipos de servicios web}

Alfonso (2012), anota que se pueden distinguir dos tipos de servicios: 
i. Servicios web SOAP (servicios web big)

Este tipo de servicio utiliza, para intercomunicarse, mensajes en lenguaje XML que siguen el estándar SOAP; estos sistemas contienen una "descripción legible por la máquina de la descripción de las operaciones ofrecidas por el servicio, escrita en WSDL [...] para definir las interfaces sintácticamente" (Alfonso, 2012, p. 6). Además, el autor destaca lo siguiente:

El formato de mensaje SOAP y el lenguaje de definición de interfaces WSDL se ha extendido bastante, y muchas herramientas de desarrollo [...] pueden reducir la complejidad de desarrollar aplicaciones de servicios Web. El diseño de un servicio basado en SOAP debe establecer un contrato formal para describir la interfaz que ofrece el servicio Web. WSDL puede utilizarse para describir los detalles del contrato (...). También deben tenerse en cuenta los requerimientos no funcionales, como por ejemplo las transacciones, necesidad de mantener el estado (addressing), seguridad y coordinación. (Alfonso, 2012, p. 6)

\section{ii. Servicios web RESTful (Representational State Transfer Web Services)}

Este tipo de servicios son "adecuados para escenarios de integración básicos adhoc", (Alfonso, 2012, p. 6). Se suelen integrar mejor con el protocolo HTTP que los servicios basados en SOAP, debido a que no requieren mensajes en lenguaje XML o de las definiciones del servicio. Estos servicios utilizan estándares como HTTP, URI, SML, MIME, y tienen una infraestructura que permite la construcción de los servicios con el mínimo uso de herramientas; así, se consigue que el desarrollo de estos servicios sea de bajo costo y de fácil adopción (Alfonso, 2012).

\subsection{Arquitectura orientada a servicios (SOA)}

En lo que se refiere a la arquitectura orientada a servicios (SOA), Conde, Del Pozo y García (2010) manifiestan lo siguiente:

La idea principal (...) de esta arquitectura es que conviene ordenar la forma en que se comunican las distintas partes de un sistema. Para conseguir este objetivo se define una capa de servicios con el que los sistemas interactúan, evitando así el trabajar de manera directa entre ellos, favoreciendo la interoperabilidad y la escalabilidad. (...). Si dos sistemas desean comunicarse, no necesitan conocer el funcionamiento del otro, sino que utilizarán esta capa de servicios como intermediaria, la cual sí conoce como funcionan estos sistemas. Si (...) se desea sustituir o realizar algún cambio en alguno de los dos sistemas, la acción sería independiente del otro, ya que se han desarrollado de manera que no dependan del otro sistema, sino (...) de los datos que devuelven. (p. 47) 
Courtois et al., (2006) indican que la SOA proporciona una metodología y un marco de trabajo para la documentación de las capacidades del negocio, y brinda soporte a las actividades de integración y consolidación.

Picón et al. (2014) subrayan que la SOA se enfoca en la organización de los procesos de negocios, y no tanto en la respectiva infraestructura tecnológica, y que gracias a su implementación brindan una ventaja competitiva a las empresas, lo cual se refleja en su rapidez de reacción ante los cambios que se presentan en los requerimientos de negocio.

Para la construcción de una arquitectura orientada a servicios se requiere de cuatro elementos esenciales:

i. Operación: Unidad de procesamiento en la arquitectura (SOA).

ii. Servicio: Contenedor de lógica, compuesto por un conjunto de operaciones que se ofrecen a sus usuarios.

iii. Mensaje: Se requiere para ejecutar una operación, para lo cual se necesitan datos de entrada, con lo que se obtienen datos de salida (resultado); estos mensajes se encargan de encapsular los datos de entrada y de salida.

iv. Proceso de negocio: Conjunto de operaciones que se ejecutan en una secuencia determinada para realizar una tarea (SOA-FUAC Wiki SOA, 20 de noviembre del 2010).

\subsubsection{Capas de software de la Arquitectura Orientada a Servicios}

En relación a las capas de la SOA, Peñaranda (2013) define las siguientes capas:

i. Aplicaciones básicas. Sistemas desarrollados en cualquier arquitectura o tecnología, bajo cualquier figura de propiedad.

ii. De exposición de funcionalidades. Las funcionalidades de la capa aplicativa se exponen en forma de servicios, en su mayoría como servicios web.

iii. De integración de servicios. Contribuyen al intercambio de datos entre los elementos de la capa aplicativa orientada a los procesos empresariales que se desarrollan en forma interna o en colaboración.

iv. De composición de procesos. Define el proceso en términos del proceso de negocio.

v. De entrega. Los servicios se despliegan a los usuarios finales. 


\section{Desarrollo de las aplicaciones web basadas en la Arquitectura Orientada \\ a Servicios (SOA)}

La metodología propuesta para desarrollar el presente trabajo fue basada en la metodología orientada a objetos con UML con la variante para SOA, dividida en las siguientes fases:

i. Identificación de servicios

ii. Clasificación de los servicios

iii. Diseño arquitectónico

iv. Construcción del servicio web

v. Implementación de interfaces de las aplicaciones web

A continuación se presenta una descripción de cada una de las fases realizadas:

\subsection{Identificación de servicios}

Este proceso consistió en la identificación de servicios, combinación de técnicas de análisis, modelos de servicio y la especificación para los servicios de negocio.

\subsubsection{Consumidor de servicios: aplicación web Sistema de Agencia de Viajes}

La figura 1 muestra el diagrama de paquetes de los procesos de negocio del Sistema de Agencia de Viajes; cada uno puede operar con diferentes aplicaciones web. En la figura 2 se presenta el diagrama de casos de uso del proceso de negocio específico para la aplicación web, que es el de gestionar reservas de hospedaje, el cual comprende los servicios de registro de clientes, reserva de habitaciones y reporte de habitaciones disponibles; es decir, requiere de servicios de otras aplicaciones y ejecuta el servicio de acuerdo a un contrato de interfaz. 
Figura 1. Diagrama de paquetes de los procesos de negocio del Sistema de Agencia de Viajes

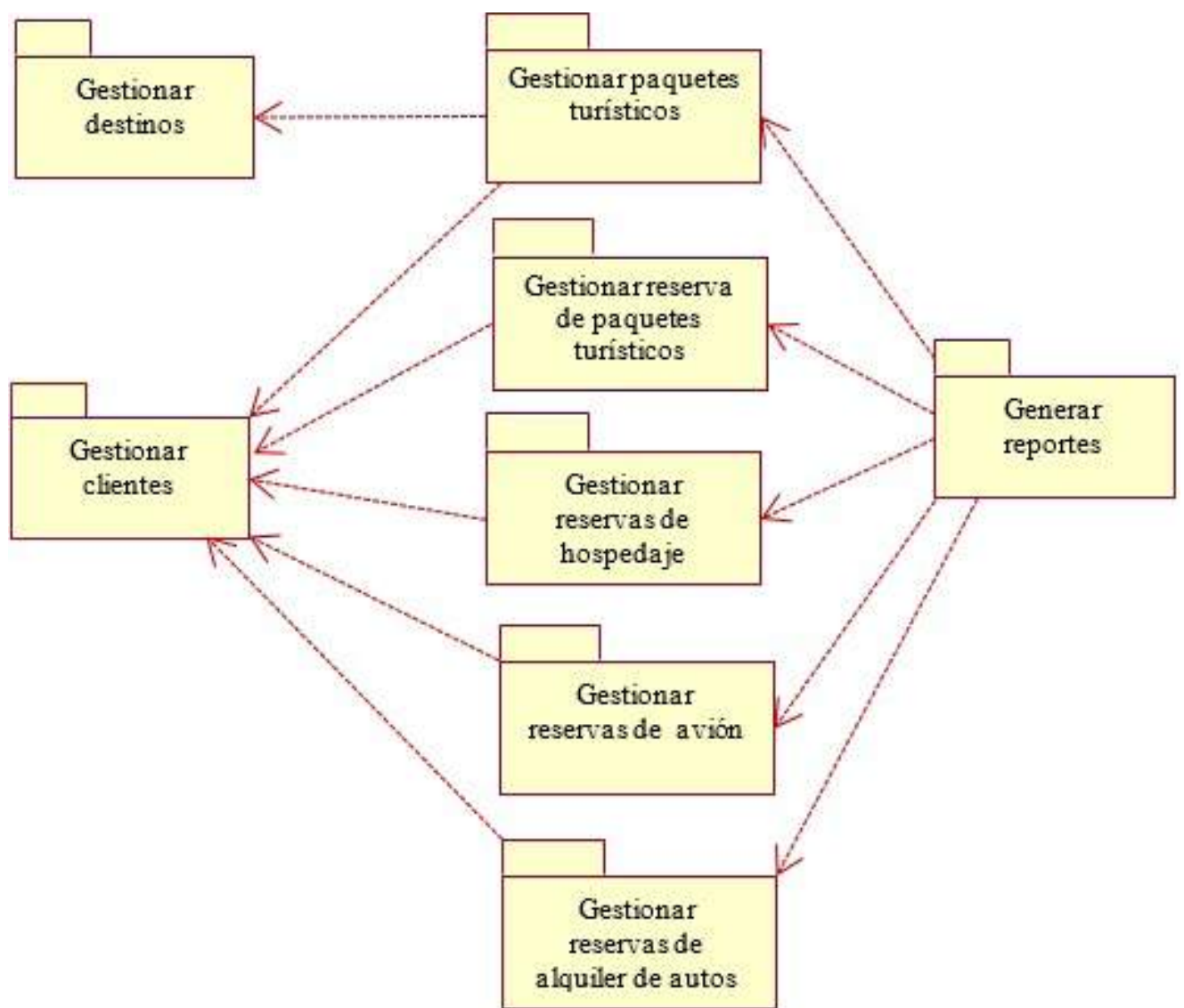

Elaboración propia 
Figura 2. Diagrama de casos de uso del paquete Gestionar Reserva de Hospedaje

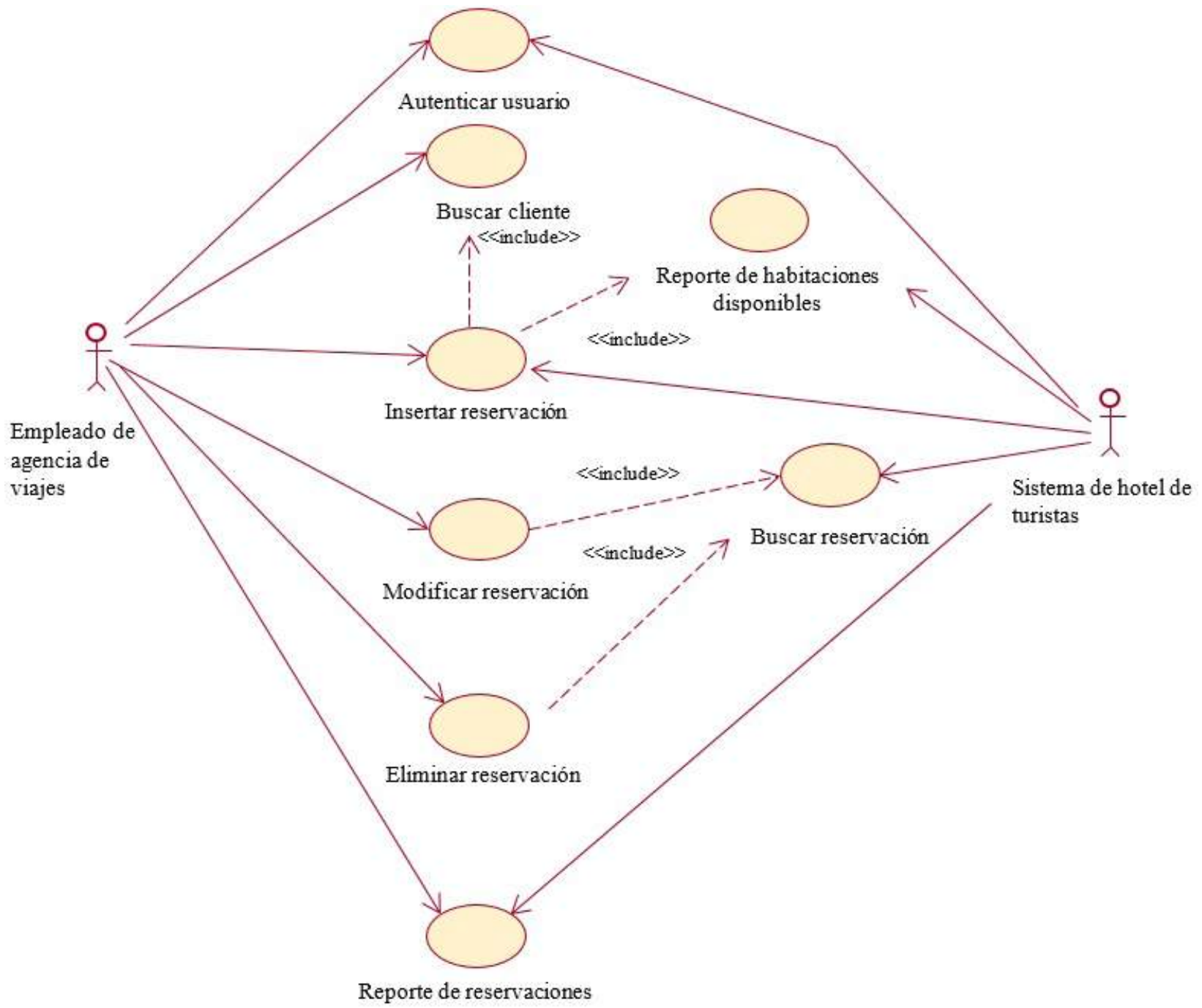

Elaboración propia 


\section{Especificación de principales casos de uso}

\begin{tabular}{|c|c|c|}
\hline \multicolumn{2}{|c|}{ Identificador } & CU-01 \\
\hline Nombre & \multicolumn{2}{|c|}{ Autenticar usuario } \\
\hline Actores & \multicolumn{2}{|c|}{ Empleado de la agencia de viajes y sistema de hotel de turistas } \\
\hline Propósito & \multicolumn{2}{|c|}{ Permitir acceso al sistema } \\
\hline Descripción & \multicolumn{2}{|c|}{$\begin{array}{l}\text { Este caso de uso empieza cuando el empleado de la agencia de viajes desea ingresar al } \\
\text { sistema }\end{array}$} \\
\hline Precondición & \multicolumn{2}{|c|}{ El empleado debe tener un usuario y contraseña } \\
\hline \multirow{4}{*}{$\begin{array}{l}\text { Secuencia } \\
\text { normal }\end{array}$} & Paso & Acción \\
\hline & 1 & $\begin{array}{l}\text { El sistema muestra el formulario de ingreso, donde se solicita el nombre de } \\
\text { usuario y la contraseña }\end{array}$ \\
\hline & 2 & $\begin{array}{l}\text { El empleado de la agencia ingresa su nombre de usuario y contraseña y } \\
\text { selecciona la opción "Aceptar" }\end{array}$ \\
\hline & 3 & $\begin{array}{l}\text { El sistema efectúa la validación de los datos del usuario e ingresa a la } \\
\text { página principal del sistema, donde se mostrarán los módulos de acuerdo } \\
\text { a los permisos }\end{array}$ \\
\hline Poscondición & \multicolumn{2}{|c|}{$\begin{array}{l}\text { El usuario accede al Sistema de Agencia de Viajes y tiene acceso al servicio de reserva de } \\
\text { hospedaje del sistema del hotel }\end{array}$} \\
\hline \multirow[t]{2}{*}{ Excepciones } & Paso & Acción \\
\hline & 2 & $\begin{array}{l}\text { Si el nombre del usuario y la contraseña no son los correctos, el sistema } \\
\text { mostrará el mensaje: "Nombre de usuario y contraseña incorrecta" }\end{array}$ \\
\hline
\end{tabular}

\begin{tabular}{|c|c|c|}
\hline \multicolumn{2}{|c|}{ Identificador } & CU-02 \\
\hline Nombre & \multicolumn{2}{|c|}{ Buscar cliente } \\
\hline Actor & \multicolumn{2}{|c|}{ Empleado de la agencia de viajes } \\
\hline Propósito & \multicolumn{2}{|c|}{ Consultar si el cliente se encuentra registrado } \\
\hline Descripción & \multicolumn{2}{|c|}{$\begin{array}{l}\text { Este caso de uso empieza cuando el empleado de la agencia consulta si el cliente se } \\
\text { encuentra registrado en el sistema }\end{array}$} \\
\hline Precondición & \multicolumn{2}{|c|}{ Tener acceso al sistema } \\
\hline \multirow[t]{2}{*}{ Secuencia } & Paso & Acción \\
\hline & 1 & El sistema muestra la interfaz de búsqueda de clientes \\
\hline \multirow{4}{*}{ normal } & 2 & $\begin{array}{l}\text { El empleado de la agencia ingresa el código y/o apellidos del cliente para } \\
\text { la búsqueda }\end{array}$ \\
\hline & 3 & El sistema muestra la lista de clientes que coincidan con la búsqueda \\
\hline & 4 & El empleado elige el cliente \\
\hline & 5 & El sistema visualiza los datos del cliente \\
\hline \multicolumn{3}{|l|}{ Poscondición } \\
\hline \multirow[t]{2}{*}{ Excepciones } & Paso & Acción \\
\hline & 2 & $\begin{array}{l}\text { Si el cliente no se encuentra registrado, se realiza el caso de uso "Gestionar } \\
\text { cliente" del sistema de reservas }\end{array}$ \\
\hline
\end{tabular}




\begin{tabular}{|c|c|c|}
\hline \multirow{2}{*}{$\begin{array}{l}\text { Identificador } \\
\text { Nombre }\end{array}$} & \multicolumn{2}{|r|}{ CU-03 } \\
\hline & \multicolumn{2}{|r|}{ Insertar reservación } \\
\hline Actores & \multicolumn{2}{|c|}{ Empleado de la agencia de viajes y sistema de hotel de turistas } \\
\hline Propósito & \multicolumn{2}{|c|}{$\begin{array}{l}\text { Registrar la reserva de hospedaje desde el Sistema de Agencia de Viajes al sistema de hotel } \\
\text { (servicio web) }\end{array}$} \\
\hline Descripción & \multicolumn{2}{|c|}{$\begin{array}{l}\text { Este caso de uso empieza cuando el empleado de la agencia de viajes realiza una reserva de } \\
\text { hospedaje en el hotel }\end{array}$} \\
\hline Precondición & \multicolumn{2}{|c|}{ El empleado de la agencia debe tener acceso al servicio de reserva de hospedaje } \\
\hline \multirow{8}{*}{$\begin{array}{l}\text { Secuencia } \\
\text { normal }\end{array}$} & Paso & Acción \\
\hline & 1 & El sistema muestra el formulario de reserva de habitaciones \\
\hline & 2 & El empleado busca al cliente \\
\hline & 3 & Se realiza el caso de uso CU-02 "Buscar cliente" \\
\hline & 4 & El empleado solicita un reporte de habitaciones disponibles \\
\hline & 5 & Se realiza el caso de uso CU-04 "Reporte de habitaciones disponibles" \\
\hline & 6 & $\begin{array}{l}\text { El empleado registra todos los datos de la reserva y selecciona la opción } \\
\text { "Agregar" }\end{array}$ \\
\hline & 7 & $\begin{array}{l}\text { El sistema efectúa la validación. Si esta fue exitosa, se muestra el mensaje: “El } \\
\text { registro de la reserva se realizó con éxito". }\end{array}$ \\
\hline Poscondición & \multicolumn{2}{|c|}{ El sistema ha grabado los datos de la reserva en el sistema del hotel a través del servicio web } \\
\hline \multirow[t]{2}{*}{ Excepciones } & Paso & Acción \\
\hline & 4 & Si no hay habitaciones disponibles del agrado del cliente, finaliza el caso de usog \\
\hline
\end{tabular}

\begin{tabular}{|l|l|l|}
\hline \multicolumn{1}{|c|}{ Identificador } & \multicolumn{2}{l|}{ CU-04 } \\
\hline Nombre & Reporte de habitaciones disponibles \\
\hline Propósito & \multicolumn{2}{l|}{ Generar un reporte de las habitaciones disponibles del sistema de hotel } \\
\hline \multirow{2}{*}{ Descripción } & $\begin{array}{l}\text { Este caso de uso empieza cuando el empleado de la agencia de solicita un reporte de las } \\
\text { habitaciones disponibles para realizar la reserva en el sistema de hotel }\end{array}$ \\
\hline \multirow{2}{*}{ Precondición } & El empleado de la agencia debe tener acceso al servicio de reserva de hospedaje \\
\hline \multirow{2}{*}{$\begin{array}{l}\text { Secuencia } \\
\text { normal }\end{array}$} & Paso & Acción \\
\cline { 2 - 3 } & 1 & El sistema muestra la interfaz de habitaciones disponibles \\
\cline { 2 - 3 } & 2 & El empleado ingresa un rango de fechas \\
\cline { 2 - 3 } & 3 & El sistema genera el reporte de habitaciones disponibles \\
\hline \multirow{2}{*}{ Poscondición } & El sistema muestra el reporte de habitaciones disponibles \\
\hline
\end{tabular}




\begin{tabular}{|c|c|c|}
\hline \multicolumn{2}{|l|}{ Identificador } & CU-05 \\
\hline Nombre & \multicolumn{2}{|c|}{ Modificar reservación } \\
\hline Actores & \multicolumn{2}{|c|}{ Empleado de la agencia de viajes y sistema de hotel de turistas } \\
\hline Propósito & \multicolumn{2}{|c|}{ Modificar la reserva de hospedaje registrada } \\
\hline Descripción & \multicolumn{2}{|c|}{$\begin{array}{l}\text { Este caso de uso empieza cuando el empleado de la agencia de viajes realiza una } \\
\text { modificación a la reserva de hospedaje en el hotel }\end{array}$} \\
\hline Precondición & \multicolumn{2}{|c|}{$\begin{array}{l}\text { El empleado de la agencia debe tener acceso al servicio de reserva de hospedaje, y para la } \\
\text { opción "Modificar" el proyecto debe encontrarse registrado }\end{array}$} \\
\hline \multirow{8}{*}{$\begin{array}{l}\text { Secuencia } \\
\text { normal }\end{array}$} & Paso & Acción \\
\hline & 1 & El empleado elige la opción "Modificar" \\
\hline & 2 & El sistema muestra la opción "Búsqueda de Reserva" \\
\hline & 3 & Se realiza el caso de uso CU-07 "Buscar reservación” \\
\hline & 4 & El empleado selecciona la reserva registrada \\
\hline & 5 & El sistema muestra el formulario con los datos registrados de la reserva \\
\hline & 6 & El empleado procede a realizar y guardar los cambios de la reserva \\
\hline & 7 & $\begin{array}{l}\text { El sistema le muestra un mensaje satisfactorio "Los cambios han sido realizados } \\
\text { con éxito" }\end{array}$ \\
\hline Poscondición & \multicolumn{2}{|c|}{ Se han actualizado los datos de la reserva } \\
\hline
\end{tabular}

\begin{tabular}{|c|c|c|}
\hline \multicolumn{2}{|l|}{ Identificador } & CU-06 \\
\hline Nombre & \multicolumn{2}{|c|}{ Eliminar reservación } \\
\hline Actores & \multicolumn{2}{|c|}{ Empleado de la agencia de viajes y sistema de hotel de turistas } \\
\hline Propósito & \multicolumn{2}{|c|}{ Eliminar la reserva de hospedaje registrada } \\
\hline Descripción & \multicolumn{2}{|c|}{ Este caso de uso empieza cuando el empleado de la agencia desea eliminar una reserva } \\
\hline Precondición & \multicolumn{2}{|c|}{$\begin{array}{l}\text { El empleado de la agencia debe tener acceso al servicio de reserva de hospedaje y para la } \\
\text { opción "Eliminar", el proyecto debe encontrarse registrado }\end{array}$} \\
\hline \multirow{10}{*}{$\begin{array}{l}\text { Secuencia } \\
\text { normal }\end{array}$} & Paso & Acción \\
\hline & 1 & El empleado elige la opción "Eliminar" \\
\hline & 2 & El sistema muestra la opción "Búsqueda de Reserva" \\
\hline & 3 & Se realiza el caso de uso CU-07 "Buscar reservación" \\
\hline & 4 & El empleado selecciona la reserva registrada \\
\hline & 5 & El sistema muestra el formulario con los datos registrados de la reserva \\
\hline & 6 & El empleado procede a eliminar la reserva \\
\hline & 7 & El sistema solicita confirmación del evento "Eliminar" \\
\hline & 8 & El empleado indica "Aceptar" \\
\hline & 9 & El sistema elimina la reserva \\
\hline Poscondición & \multicolumn{2}{|c|}{ Se ha eliminado la reserva registrada } \\
\hline
\end{tabular}


De igual forma, se especificaron los siguientes casos de uso: CU-07 Buscar reservación y CU-08 Reporte de reservaciones.

\subsubsection{Proveedor de servicios: aplicación web del sistema de reservas de hospedaje}

Una de las características más resaltantes de SOA es que está basada en contratos, donde el proveedor establece las reglas de comunicación, el transporte y los datos de entrada y salida que serán intercambiados por ambas partes.

El sistema de reserva de hospedaje a través de la red acepta y ejecuta consultas de los consumidores; además publica sus servicios y su contrato de interfaces en el registro de servicios para que el consumidor de servicios pueda descubrir y acceder al servicio.

Es importante precisar que los procesos de negocio identificados en el sistema de reserva de hospedaje son los mismos que hemos identificado en el Sistema de Agencia de Viajes, por lo que es en este sistema que se implementan los servicios web.

En la tabla 1 se describen los procesos de negocio identificados como servicios del sistema de reservas de hospedaje de un hotel. El funcionamiento del sistema está en función a la actividad principal (hospedaje), permitiendo que el sistema lleve un control de las reservas y registros de hospedaje de los clientes. La figura 3 muestra el diagrama de casos de uso del sistema de reservas de hospedaje de un hotel.

Tabla 1. Procesos de negocio del sistema de reservas de hotel

\begin{tabular}{l|l}
\hline \multicolumn{1}{c|}{ Procesos de negocio } & \multicolumn{1}{c}{ Descripción } \\
\hline Gestionar cliente & $\begin{array}{l}\text { Este proceso se encarga de gestionar los clientes de la agencia, como hacer la } \\
\text { transferencia de clientes a los proveedores. }\end{array}$ \\
\hline $\begin{array}{l}\text { Gestionar reservas de } \\
\text { hospedaje }\end{array}$ & $\begin{array}{l}\text { Este proceso representa la gestión que lleva a cabo la agencia de viajes para realizar } \\
\text { las reservaciones de las habitaciones que se encuentran disponibles en un hotel. }\end{array}$ \\
\hline Gestionar reportes & $\begin{array}{l}\text { Este proceso representa la obtención de información que se genera en cada } \\
\text { gestión para la toma de decisiones por parte de la agencia. }\end{array}$ \\
\hline
\end{tabular}


Figura 3. Diagrama de casos de uso del sistema de reserva de hospedaje de un hotel

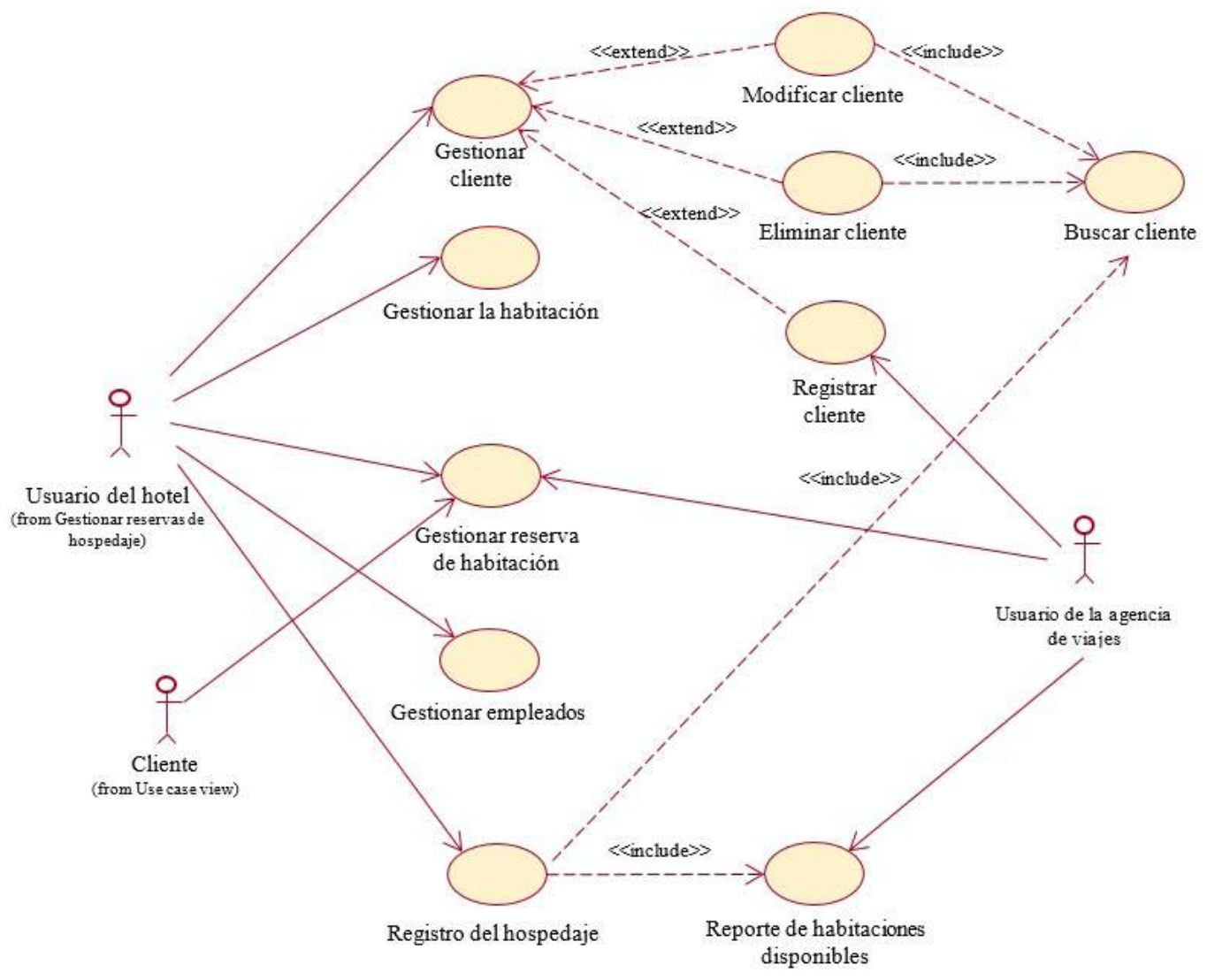

Elaboración propia 


\section{Especificación de principales casos de uso}

\begin{tabular}{|c|c|c|}
\hline \multicolumn{2}{|c|}{ Identificador } & CU-01 \\
\hline Nombre & \multicolumn{2}{|c|}{ Gestionar clientes } \\
\hline Actores & \multicolumn{2}{|c|}{ Usuario del hotel y usuario de la agencia de viajes } \\
\hline Propósito & \multicolumn{2}{|c|}{$\begin{array}{l}\text { Permite a los usuarios gestionar los datos del cliente. Esto incluye registrar, modificar y } \\
\text { eliminar }\end{array}$} \\
\hline Descripción & \multicolumn{2}{|c|}{ Este caso de comienza cuando el usuario solicita iniciar el proceso de gestionar clientes } \\
\hline Precondición & \multicolumn{2}{|c|}{$\begin{array}{l}\text { El usuario debe tener acceso al sistema y los permisos correspondiente para registrar, } \\
\text { modificar y eliminar }\end{array}$} \\
\hline \multirow{23}{*}{$\begin{array}{l}\text { Secuencia } \\
\text { normal }\end{array}$} & Paso & Acción \\
\hline & 1 & El usuario elige la opción "Gestionar clientes" \\
\hline & 2 & El sistema muestra la interfaz de gestionar clientes \\
\hline & 3 & El empleado selecciona el proceso de búsqueda \\
\hline & 4 & Se realiza el caso de uso "Buscar cliente" \\
\hline & 5 & $\begin{array}{l}\text { El empleado elige la opción requerida en el sistema, ver subflujos de } \\
\text { registrar, eliminar y modificar clientes }\end{array}$ \\
\hline & \multicolumn{2}{|c|}{ Subflujo: $<<$ Registrar cliente $>>$} \\
\hline & 1 & El usuario elige la opción "Agregar" \\
\hline & 2 & El sistema muestra la interfaz de registro de cliente \\
\hline & 3 & El usuario registra y guarda los datos del cliente \\
\hline & 4 & $\begin{array}{l}\text { El sistema efectúa la validación de los datos. Si esta fue conforme, se muestra } \\
\text { el mensaje: "El registro del cliente se realizó con éxito" }\end{array}$ \\
\hline & 5 & Finaliza el caso de uso \\
\hline & \multicolumn{2}{|c|}{ Subflujo: <<Modificar cliente>> } \\
\hline & 1 & El usuario selecciona al cliente a modificar. \\
\hline & 2 & El sistema muestra los datos del cliente y elige la opción "Modificar". \\
\hline & 3 & El usuario actualiza los datos del cliente y graba. \\
\hline & 4 & $\begin{array}{l}\text { El sistema le muestra un mensaje: "Los cambios han sido realizados con } \\
\text { éxito". }\end{array}$ \\
\hline & 5 & Finaliza el caso de uso. \\
\hline & \multicolumn{2}{|c|}{ Subflujo: <<Eliminar cliente >> } \\
\hline & 1 & El usuario selecciona el cliente a eliminar y elige la opción "Eliminar" \\
\hline & 2 & El sistema solicita confirmación del evento "Eliminar" \\
\hline & 3 & El usuario indica "Aceptar" \\
\hline & 4 & El sistema elimina el cliente y finaliza el caso de uso \\
\hline Poscondición & \multicolumn{2}{|c|}{ Los datos del cliente quedaron grabados en el sistema } \\
\hline
\end{tabular}




\begin{tabular}{|c|c|c|}
\hline \multicolumn{2}{|c|}{ Identificador } & CU-05 \\
\hline Nombre & \multicolumn{2}{|c|}{ Registro de hospedaje } \\
\hline Actores & \multicolumn{2}{|c|}{ Usuario de hotel } \\
\hline Propósito & \multicolumn{2}{|c|}{$\begin{array}{l}\text { Registrar el registro de hospedaje para aquellos clientes que no han realizado una } \\
\text { reserva }\end{array}$} \\
\hline Descripción & \multicolumn{2}{|c|}{$\begin{array}{l}\text { El caso de uso comienza cuando el usuario del hotel registra un hospedaje sin tener una } \\
\text { reserva previa }\end{array}$} \\
\hline Precondición & \multicolumn{2}{|c|}{ El usuario del hotel debe tener acceso al sistema } \\
\hline \multirow{10}{*}{$\begin{array}{l}\text { Secuencia } \\
\text { normal }\end{array}$} & Paso & Acción \\
\hline & 1 & El usuario elige la opción "Registrar Hospedaje" \\
\hline & 2 & El sistema muestra la interfaz de registro de hospedaje \\
\hline & 3 & El usuario busca al cliente \\
\hline & 4 & Se realiza el caso de uso CU-07 "Buscar cliente" \\
\hline & 5 & El sistema muestra los datos del cliente \\
\hline & 6 & El usuario genera un reporte de habitaciones disponibles \\
\hline & 7 & Se realiza el caso de uso CU-06 "Reporte de habitaciones disponibles" \\
\hline & 8 & $\begin{array}{l}\text { El empleado registra todos los datos del registro de hospedaje y selecciona } \\
\text { la opción "Agregar" }\end{array}$ \\
\hline & 9 & $\begin{array}{l}\text { El sistema efectúa la validación los datos. Si esta fue exitosa, se muestra un } \\
\text { mensaje satisfactorio: "El registro de hospedaje se realizó con éxito" }\end{array}$ \\
\hline Poscondición & \multicolumn{2}{|c|}{ Se ha almacenado el registro de hospedaje } \\
\hline
\end{tabular}

De igual forma, se especificaron los siguientes casos de uso: CU-02 Gestionar la habitación, CU-04 Gestionar empleados, CU-07 Buscar cliente. El caso de uso CU-03 Gestionar reserva de habitación y CU-06 Reporte de habitaciones disponibles son las mismas que se describen en el diagrama de casos de uso Gestionar reserva de hospedaje del Sistema de Agencia de Viajes por ser el mismo servicio que se ofrece (figura 2).

\subsection{Clasificación de los servicios}

Esta actividad empieza una vez que se han identificado los servicios y definidos los contratos. Los contratos de servicios entre el consumidor y proveedor son los siguientes:

i. Contrato de servicio registro de clientes: Este servicio tiene la finalidad de registrar a los clientes de la agencia de viajes como clientes del hotel. La forma de uso es registrarse primero como cliente de la aplicación web de la agencia de viaje, y luego se transfiere para el registro correspondiente en la aplicación web del hotel. La restricción que tiene este servicio es que solo permite el registro; no puede modificar ni eliminar cuando ya es cliente del hotel. 
ii. Contrato de servicio de reservación de habitación: Este servicio tiene la finalidad de realizar reservaciones de habitaciones con la aplicación web del hotel desde la aplicación web agencia de viajes que ofrece el mismo servicio. Respecto a las restricciones, no puede realizar una reservación de una habitación ocupada.

iii. Contrato del servicio reportes: Este servicio tiene la finalidad de mostrar los siguientes reportes: habitaciones disponibles y reservaciones realizadas. No tiene restricciones.

\subsection{Diseño arquitectónico}

El diseño arquitectónico es un proceso en el que se intenta establecer una organización del sistema que permita satisfacer los requerimientos de este. La arquitectura del sistema puede basarse en un modelo o estilo arquitectónico eligiendo la estructura más adecuada. Existen distintos tipos de arquitectura:

i. Arquitectura monolítica. Arquitectura rígida de programación en un solo computador.

ii. Arquitectura Cliente/Servidor. Arquitectura que consiste en un conjunto de servicios proporcionados por los servidores y un conjunto de clientes que acceden y usan dichos servicios (Sommerville, 2011).

iii. Arquitectura de tres capas. Es una especialización de la arquitectura Cliente Servidor, donde las funciones de la aplicación se dividen en capa de presentación, capa de lógica de negocio y capa de datos.

La solución propuesta en la investigación está basada en una arquitectura de tres capas: la capa de presentación, la capa de la lógica de negocios y la capa de datos, porque permite utilizar de mejor manera los recursos del servicio web. Asimismo, este tipo de arquitectura presenta mayor flexibilidad para realizar el mantenimiento por capas y mejor disposición para escalar a futuro.

i. Capa de presentación. Es la interfaz que se muestra al usuario final de la aplicación. Funciona en cualquier computadora con conexión a Internet. La conexión a Internet es necesaria para obtener información del servidor a través de una petición SOAP.

ii. Capa lógica de negocios. Maneja las transacciones según la lógica del sistema y contacta al servidor de base de datos a través de consultas SQL. El servidor SOAP responde a las peticiones de las computadoras y es capaz de generar respuestas en formato cadena con estructura XML.

iii. Capa de datos. El servidor de base de datos está encargado de guardar la información detallada de las reservas de paquetes turísticos, información de los 
destinos y paquetes que ofrece la agencia de viajes, de los clientes, y sobre estos se generan las consultas. Utiliza el motor de base de datos SQL Server 2014. En este contexto responde a las peticiones de los servicios web.

\subsubsection{Arquitectura física}

En las figuras 4 y 5 se muestra la arquitectura concreta de servicios de negocio de la aplicación web del Sistema de Agencia de Viajes y del Sistema de Reservas de Hospedaje con sus principales capas involucradas en el funcionamiento del sistema, y sus interrelaciones.

Figura 4. Arquitectura física de la aplicación web del Sistema Agencia de Viajes

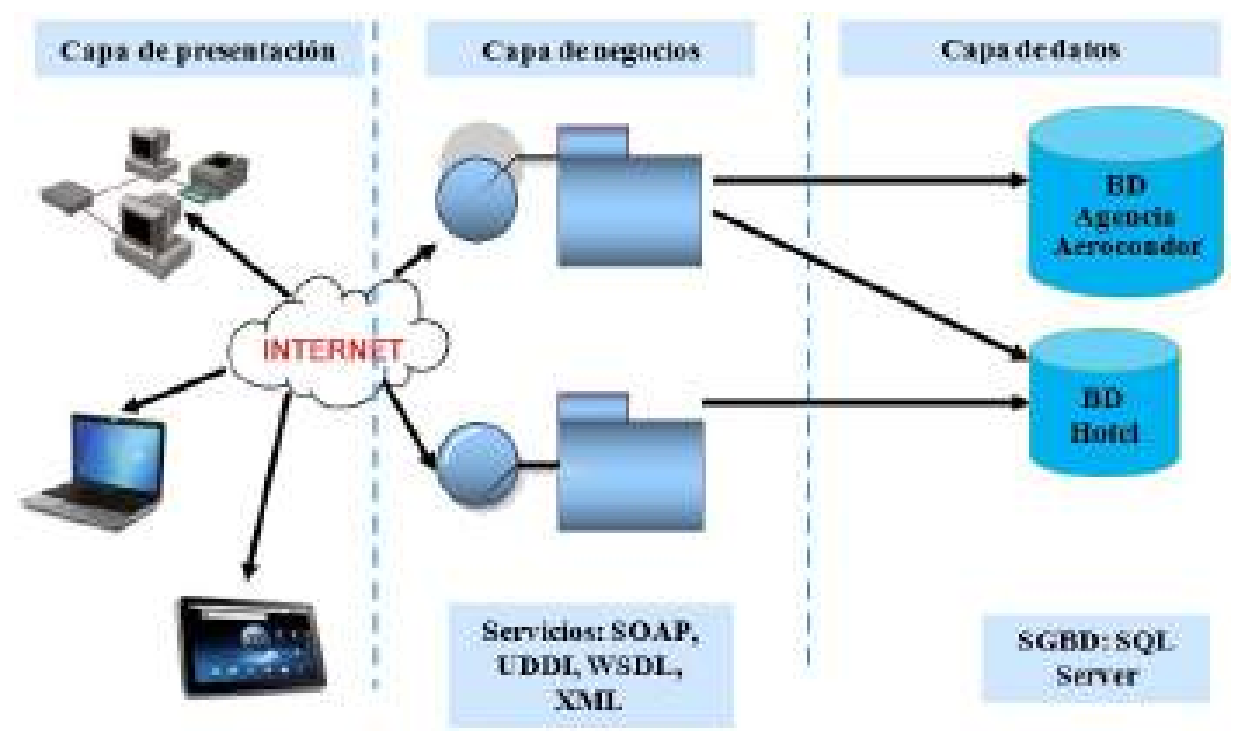

Elaboración propia 
Figura 5. Arquitectura física de la aplicación web del Sistema de Reserva de Hotel

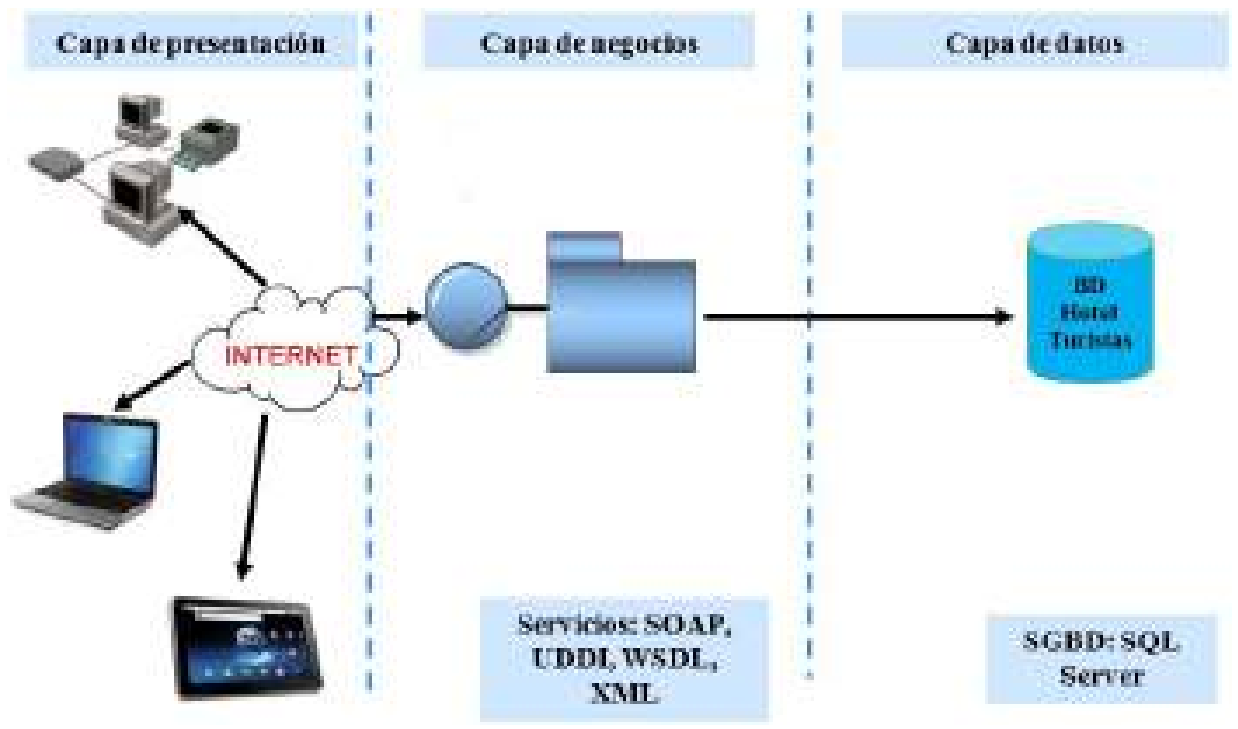

Elaboración propia

\subsubsection{Arquitectura lógica}

La figura 6 muestra la arquitectura lógica de la aplicación web Sistema de Agencia de Viajes. La aplicación está dividida por subsistemas o módulos que responden a todo el funcionamiento del sistema.

Figura 6. Arquitectura lógica de la aplicación web del Sistema Agencia de Viajes

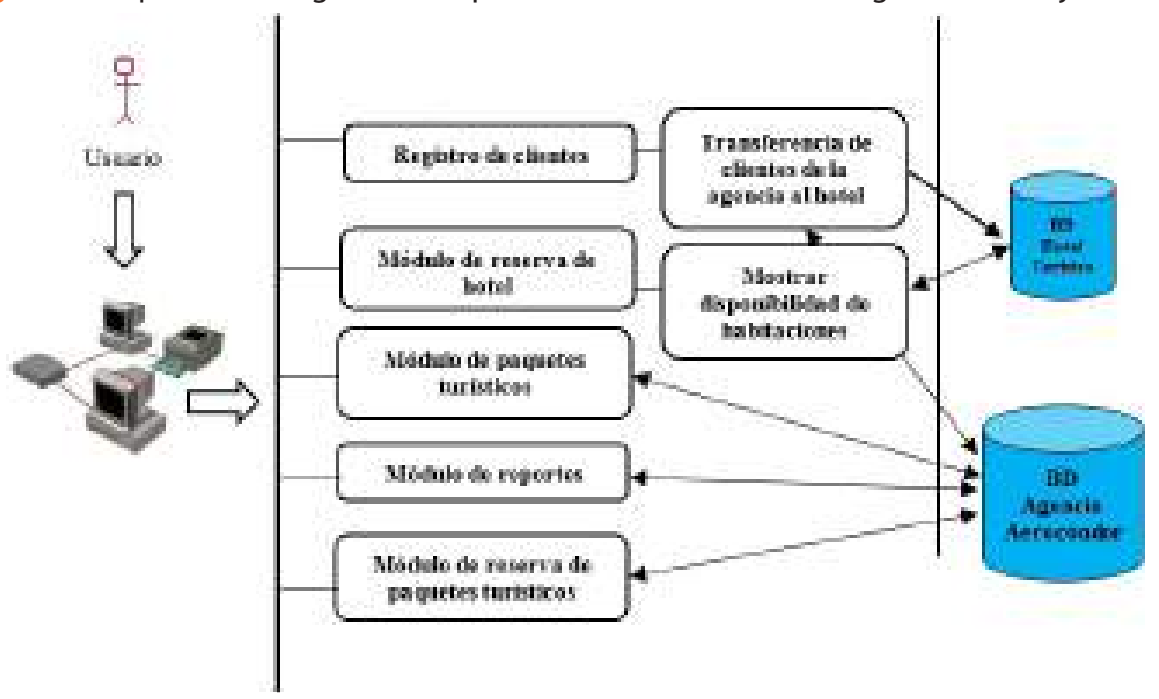

Elaboración propia 


\subsubsection{Diseño de la base de datos}

Las figuras 7 y 8 muestran el diseño de las bases de datos de las aplicaciones web representado por el diagrama Entidad-Relación.

Figura 7. Modelo lógico de datos del Sistema de Agencia de Viajes

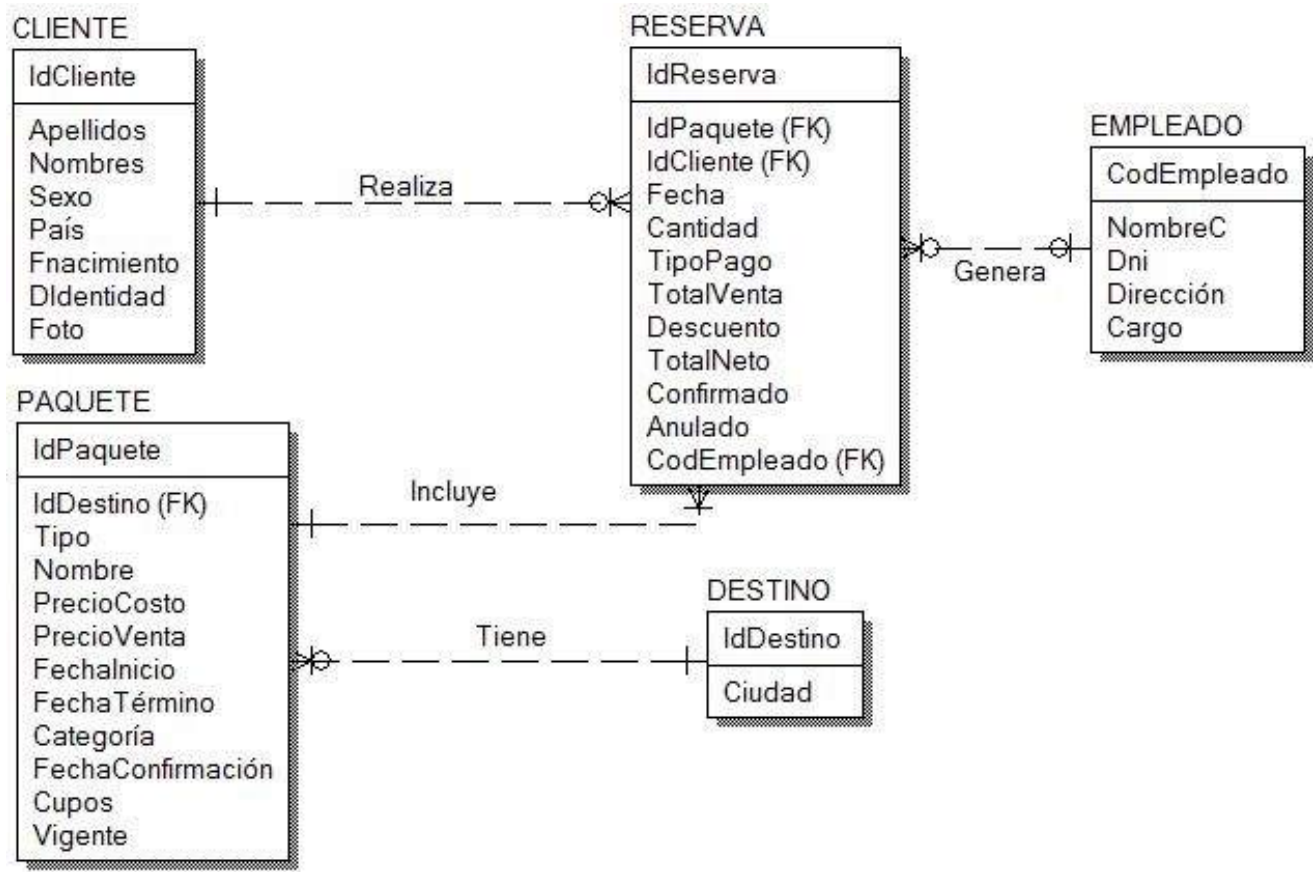

Elaboración propia 
Figura 8. Modelo lógico de datos del Sistema de hotel

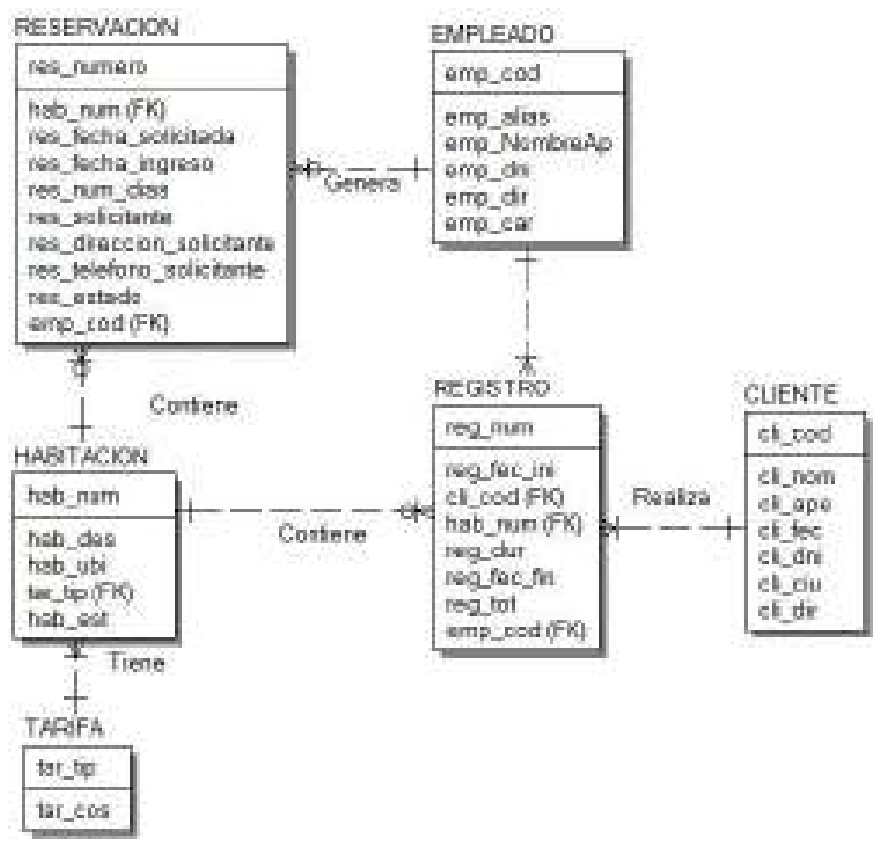

Elaboración propia

\subsection{Construcción del servicio web}

Los servicios web sirven para intercambiar información entre distintas aplicaciones computacionales en la web, y poseen componentes estandarizados para describir su funcionamiento a través de capas de especificaciones; así logran una alta interoperabilidad y una eficiente integración entre los distintos sistemas heterogéneos y distribuidos en la web (Ochoa et al., 2009).

Un servicio web se define como un componente al que se accede mediante protocolos Web estándar; utiliza XML para el intercambio de mensajes. XML asegura la interoperabilidad entre las aplicaciones, ya que tiene la capacidad de ser transportado a través de la mayoría de protocolos de la web.

En el ámbito de las pymes se trata de identificar los servicios que proveen la funcionalidad e información que requiere el proceso de negocio y hacerlos disponibles en forma de servicio web para poder acceder a estos por dicho proceso de forma uniforme y completa.

El servicio web que se implementó, WSMasterHotel, es el servicio web que se utiliza y se encarga de gestionar los servicios de registro de clientes, reserva de habitaciones y generar reportes. Para cada servicio identificado se realiza el contrato funcional con 
WSDL, es decir, se define la interfaz pública que establece la manera en que el servicio web se comunicará con el proceso de negocio u otros servicios web.

La figura 9 muestra el modelo de despliegue del servicio web que distribuyen los diversos elementos de la arquitectura orientada a servicios. La aplicación web de la agencia de viajes solicita información sobre la disponibilidad de habitaciones para poder realizar una reserva de habitación, haciendo una petición a la aplicación web de hotel de sus servicios a través de internet. En todo este proceso intervienen una serie de estándares web que hacen posible esta circulación de información. Estas son: XML, SOAP, WSDL y UDDI.

Figura 9. Modelo de despliegue del servicio web con SOA

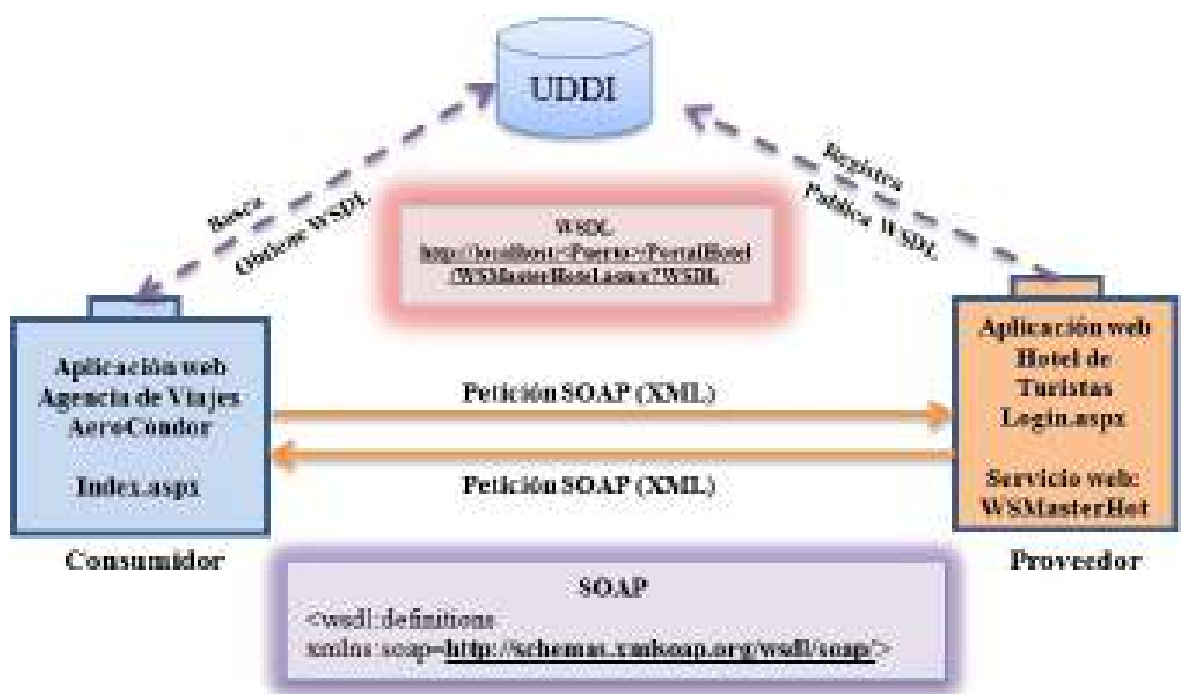

Elaboración propia

\subsection{Implementación de interfaces de las aplicaciones web}

Las figuras 10, 11, 12 y 13 presentan algunas de las interfaces implementadas en las aplicaciones web del Sistema de Agencia de viajes y el Sistema de Reserva de hospedaje de un hotel. 
Figura 10. Interfaz del portal web de la agencia de viajes AeroCóndor

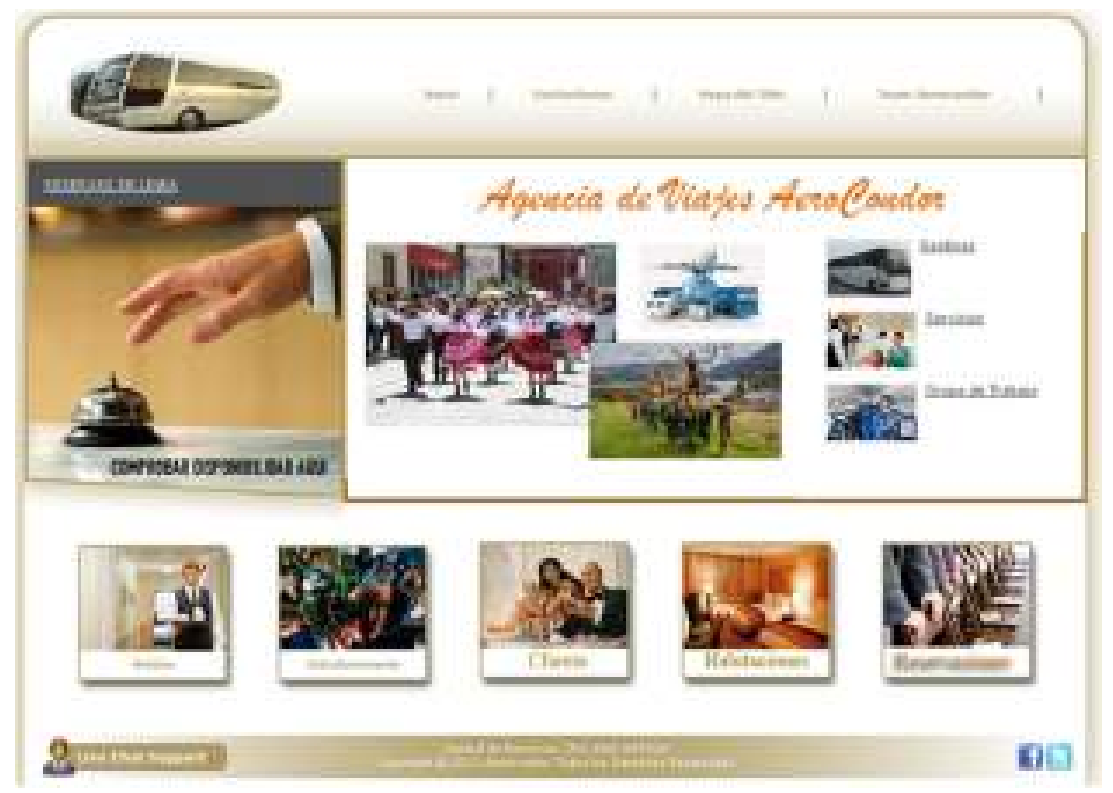

Elaboración propia

Figura 11. Interfaz de contrato del servicio: reservación de habitaciones del Hotel de Turistas

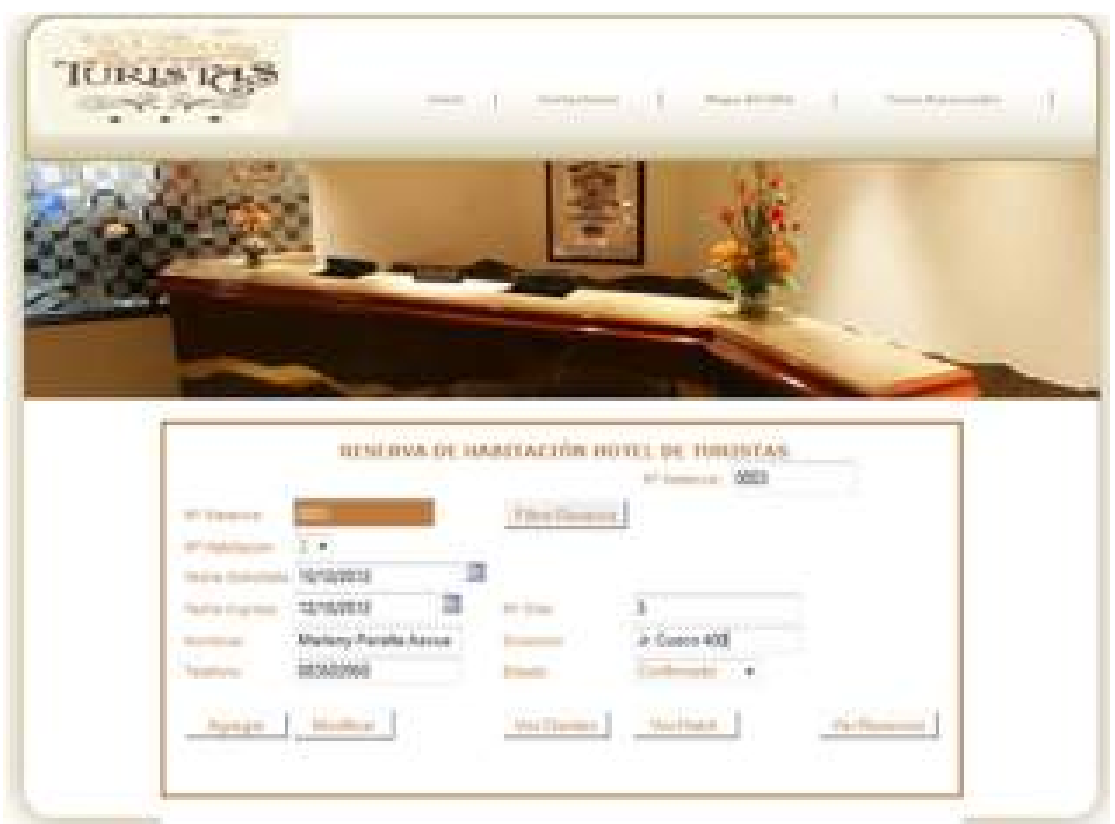

Elaboración propia 
Figura 12. Interfaz del portal web del Hotel Turistas

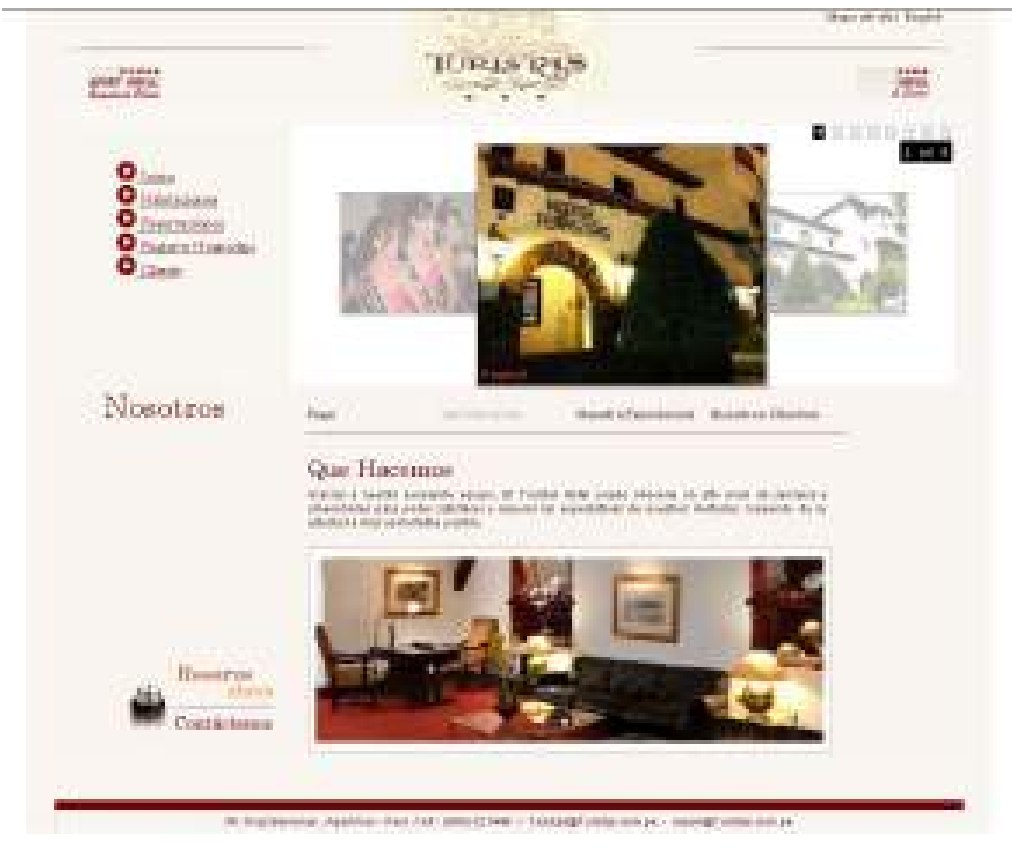

Elaboración propia

Figura 13. Interfaz del servicio de reservación del Hotel Turistas

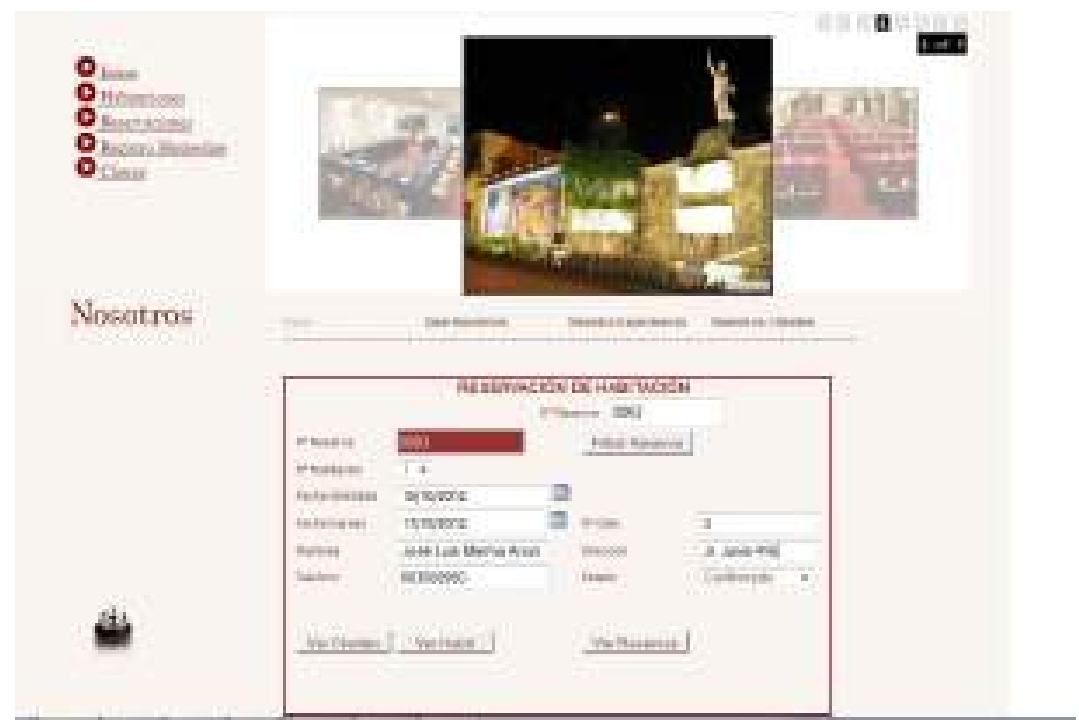

Elaboración propia 


\section{Tecnologías utilizadas}

i. Herramientas de programación

Las herramientas utilizadas fueron las siguientes:

- Microsoft Visual Studio - Es un entorno de desarrollo integrado (IDE, por sus siglas en inglés) para sistemas operativos Windows. Soporta varios lenguajes de programación tales como Visual C\# y ASP.NET.

- JavaScript, ajax, css - Permite a los desarrolladores crear acciones en las páginas web.

ii. Servicios web

SOAP (Simple Object Access Protocol) - Es un protocolo estándar que define cómo dos objetos en diferentes procesos pueden comunicarse por medio de intercambio de datos XML. Es uno de los protocolos utilizados en los servicios Web.

iii. Base de datos

SQLServer 2012 - Es un sistema de gestión de bases de datos relacional y multiusuario. La aplicación web desarrollada puede interactuar con cualquier tipo de base de datos, siempre que se disponga del conector de esta.

iv. Herramientas CASE

- Rational Rose vr 7.0.0 - Herramienta utilizada para el modelado visual mediante UML, que permite especificar, analizar y diseñar antes de codificarlo.

- AllFusion Erwin Data Modeler 7.2 - Herramienta utilizada para el modelamiento de la base de datos.

\section{Resultados y discusión}

En el presente trabajo se comprobó la integración y comunicación interempresarial mediante aplicaciones web basadas en la Arquitectura Orientada a Servicios (SOA). Con la implementación del servicio web se lograron ciertos beneficios, como la automatización de consultas definidas y la información accesible para la gestión de reservas en un hotel desde una agencia de viajes.

Los servicios web proporcionan mecanismos que posibilitaron a las aplicaciones comunicarse entre sí. Se empaquetaron las aplicaciones de software en servicios reusables con interfaces estándares e invocables que puedan ser llamadas en secuencias definidas para 
formar procesos de negocios. Así, se utilizaron mecanismos estandarizados para apoyar la comunicación entre los servicios y la capacidad de combinar servicios para soportar funcionalidades complejas. La figura 14 muestra el esquema que representa el resultado final de la implementación, en la que dos o más empresas pueden integrarse y compartir servicios utilizando la tecnología orientada a servicios (SOA).

Figura 14. SOA en pequeñas empresas

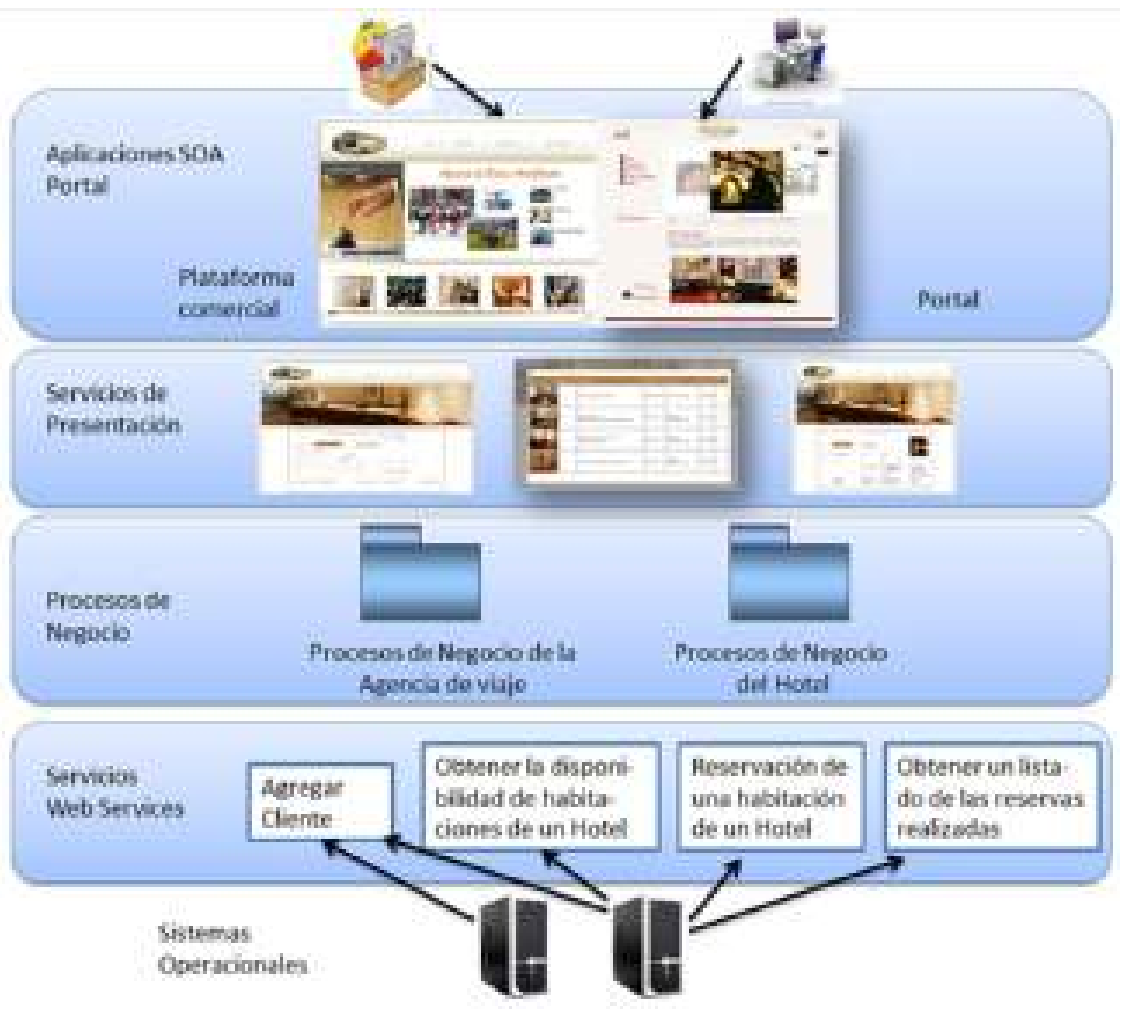

Elaboración propia

\section{Conclusiones y recomendaciones}

Este trabajo permitió demostrar que a través de aplicaciones web y servicio web bajo la Arquitectura Orientada a Servicios (SOA) fue posible establecer la integración y comunicación entre dos empresas. El servicio web implementado de reservaciones de habitaciones del Hotel de Turistas puede ser reutilizado para integrar más empresas dedicadas a este mismo rubro como hostales, hoteles, residencias, etcétera. Esto hace factible la reutilización de servicios para la creación de nuevas aplicaciones o funcionalidades que apoyen los procesos de negocio. Asimismo, se evidenció que el servicio web es la implementación de una actividad dentro de un proceso de negocio, y que un proceso, a su vez, se exterioriza como servicio web. 
Se lograron definir los procesos de negocio como servicios, lo que permitió establecer los contratos de servicio entre el proveedor y el consumidor. Por ello, es importante una buena definición de los objetivos por conseguir mediante el uso de la arquitectura SOA y determinar con claridad los contratos de servicios por crear, así como el acceso a los servicios y datos. La construcción del servicio web establece un gran apoyo en el desarrollo de los procesos de negocio aumentando su funcionalidad y valor global del negocio.

Mediante el desarrollo de aplicaciones web basadas en la Arquitectura Orientada a Servicios (SOA) se demuestra que la arquitectura representa un cambio en el modelo de diseño, de programación y de despliegue. Las empresas que responden al modelo de la SOA obtendrán múltiples beneficios, entre ellos mejor disposición para responder al cambio tecnológico, mayor interoperabilidad entre diferentes sistemas y reutilización de las funcionalidades a un nivel superior, en lugar de la tradicional reutilización de código.

Se recomienda profundizar la investigación en el estudio de los servicios web y las arquitecturas orientadas a servicios. De esa manera se posibilitará evolucionar la propuesta de versiones futuras incorporándole elementos tan significativos como el uso de patrones de integración empresarial y la aplicación de Servicios Web RESTful (REpresentational State Transfer Web Services), para poder realizar las comparaciones de rendimiento.

\section{Referencias}

Alfonso, M. (2012). Apuntes de clase. Servicios Web y SOA. Universidad de Alicante, España. Recuperado de http://www.jtech.ua.es/j2ee/publico/servc-web-2012-13/wholesite.pdf

Bazán, P. (2009). Un modelo de integrabilidad con SOA y BPM (Tesis de maestría). Universidad Nacional de La Plata, Argentina. Recuperado de http://sedici.unlp.edu.ar/ bitstream/ handle/10915/4181/Documento_completo.pdf?sequence=1

Caicedo, S., Bustos, L., y Rojas, J. (2008). Integración de procesos utilizando la Arquitectura Orientada a Servicios - SOA. Scientia et Technica, 3(40), 177-182. Recuperado de http://revistas.utp.edu.co/index.php/revistaciencia/article/download/3093/1693

Conde, M., Del Pozo, A., y García, F. (2010). Moodle 2.0 y las nuevas plataformas de aprendizaje orientadas a servicios. En Actas de los Talleres de las Jornadas de Ingeniería del Software y Bases de Datos, 4(3), 45-56. Recuperado de https://repositorio.grial.eu/ bitstream/ grial/582/1/versionfinal.pdf

Courtois, G., Telmo, P., Sosto, A., Bordón, M., Gargiulo, D., Lapera, M., y Colonna, E. (2006). Modelo de Sistema para la Gestión de Pacientes en Hospitales Públicos bajo arquitectura SOA. En 
VIII Workshop de Investigadores en Ciencias de la Computación. Recuperado de http://sedici. unlp.edu.ar/bitstream/handle/10915/20807/Documento_completo.pdf?sequence=1

López, G., Echevarría, A., Fierro, P., y Jeder, I. (2007). Una propuesta de modelos de ciclo de vida (MCVS) para la integración de los procesos de negocio utilizando Service Oriented Architecture (SOA). En IX Workshop de Investigadores en Ciencias de la Computación (559-563). Chubut, Argentina: Universidad Nacional de la Patagonia San Juan Bosco. Recuperado de http://sedici.unlp.edu. ar/bitstream/handle/10915/20458/Documento_completo.pdf?sequence=1

Ochoa, S., Bastarrica, C., y Gutiérrez, C. (Eds.). (2009). Documentación electrónica e interoperabilidad de la información. Santiago de Chile: Imprenta Maval Ltda. Recuperado de https://www. dcc.uchile.cl/docs/Libro_interoperabilidad.pdfPeñaranda, J. (2013). Estandarización e interoperabilidad. Recuperado de http://www.managementensalud.com.ar/OPS_TomasSandor/ Interoperabilidad_SOA.doc

Picón, D., Fontana, F., y Martín, A. (2014). Integración de Procesos de Negocio aplicando Servicios Web. En Informe Científico Técnico UNPA, 6(2), 57-89. Recuperado de https://dialnet.unirioja. es/descarga/articulo/5123616.pdf

San José, J. (2016). Propuesta de arquitectura basada en servicios web y agentes para el desarrollo de aplicaciones de seguimiento y trazabilidad de productos (Tesis doctoral). Universidad de Castilla - La Mancha, España. Recuperado de https://ruidera.uclm.es/xmlui/handle/10578/10272

SOA - FUAC Wiki SOA. (20 de noviembre de 2010). SOA Conceptos. Recuperado de http://soafuac. wikidot.com/example-item-2

Sommerville, I. (2011). Ingeniería de software (9. a ed.). México: Addison Wesley.

Veintimilla, J., Espinoza, M., y Cisneros, F. (2014). Estudio y selección de una arquitectura orientada a servicios (SOA) que permita la integración de sistemas informáticos legados. Revista Tecnológica ESPOL-RTE, 27(1), 14-21. Recuperado de http://www.rte.espol.edu.ec/index. php/tecnologica/article/download/297/200 
